
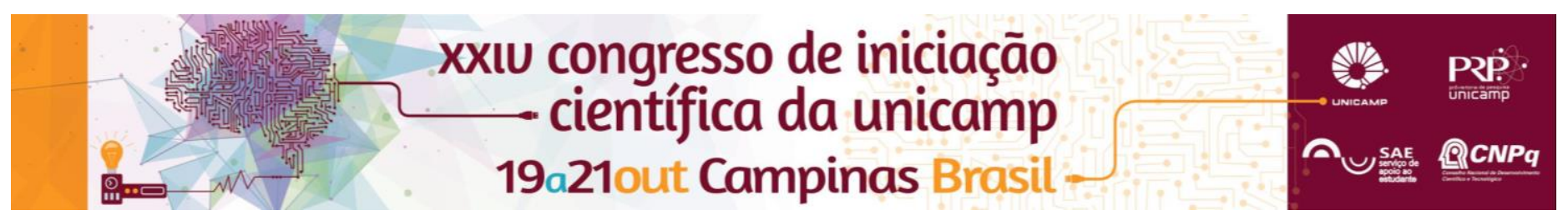

\title{
The bile acid TUDCA modulates insulin clearance
}

\author{
Luciana de Souza L. Michelone*, Vanessa Yumi Nakanishi, Jean F. Vettorazzi, Jose Maria C. Junior, Miriam A. \\ Kurauti, Antonio C. Boschero, Everardo M. Carneiro
}

\begin{abstract}
Obesity and type 2 diabetes affects million peoples around the world, and is characterized by hyperinsulinemia state which impairs many tissues function and insulin action. The hyperinsulinemic is due to enhanced insulin secretion by pancreatic islets and a reduced liver insulin clearance and IDE expression. The Tauroursodeoxycholic acid is secreted mainly after meal ingestion and have an important function in digestion. Previous studies have been demonstrated that TUDCA improves glucose tolerance, insulin sensitivity and insulin secretion in insulin resistant models. However the effect of this bile acid on insulin clearance have not been explored yet. Here, we acessed insulin clearance and liver IDE expression in C57BL6 mice fed high fat diet (HFD) and treated with TUDCA (HFD + TUDCA). As expected the mice fed on HFD presents impaired glucose tolerance, insulin sensitivity, and increased insulinemia and c-peptide, and reduction in insulin clearance and IDE expression. The impairments of HFD upon glucose tolerance, insulin sensitivity, and insulin clearance were improved by TUDCA administration. Interestingly, the reestablishment of insulinemia in HFD + TUDCA was associated with an augmented IDE expression on liver. So, we conclude that the bile acid TUDCA, in addition to regulates insulin secretion and signaling in HFD fed mice, also recovery the plasma insulin levels probably due to an increased in IDE expression in the liver which also may contributes to the improvement of glucose homeostasis.
\end{abstract}

Key words: Hyperinsulinemia, insulin clearance, TUDCA

\section{Introduction}

Obesity and type 2 diabetes is associated with insulin resistance and hyperinsulinemia, which impairs insulin receptor function and increase insulin resistance frame. Two process regulates the insulin plasm concentration: insulin secretion and insulin clearance, the last one occurs in the liver where $75 \%$ of the secreted insulin is degraded by the IDE (insulin degrading enzyme). The bile acid Tauroursodeoxycholic (TUDCA) is synthetized into the liver, has a known function in the digestion process and also modulates glucose homeostasis improving insulin resistance and increases insulin secretion in pancreatic $\beta$ cells. However, the effect of TUDCA on insulin clearance is still unknown. Here, we investigate the possible modulation of IDE by TUDCA.

\section{Results and Discussion}

C57BL6 mice were fed or not on a high fat diet (HFD $X$ $\mathrm{CON}$, respectively), and treated or not with $300 \mathrm{mg} / \mathrm{kg}$ TUDCA, i.p, for 15 days (CON TUDCA X HFD TUDCA). Glucose homeostasis were accessed by glucose and insulin tolerance test (GTT and ITT, respectively). Blood samples were collected during GTT and in fed state, and insulin and c-peptide measured by elisa. The cpeptide/insulin ratio was calculated as a functional parameter of insulin clearance. IDE liver expression was accessed by Western Blot. As expected HFD induced hyperinsulinemia, glucose intolerance, insulin resistance, and reduced insulin clearance which was associated with reduced IDE expression in the liver. The HFD+TUDCA mice exhibit improved of insulin and glucose tolerance. Interestingly, the TUDCA treated mice fed on HFD also reduced c-peptide/insulin ratio, suggesting an augmented insulin clearance which seems occurred due to an increase in IDE expression in the liver and may contributes, at least in part, to the recovery of plasmatic insulin levels of HFD fed mice. The CON + TUDCA mice do not present any alterations.
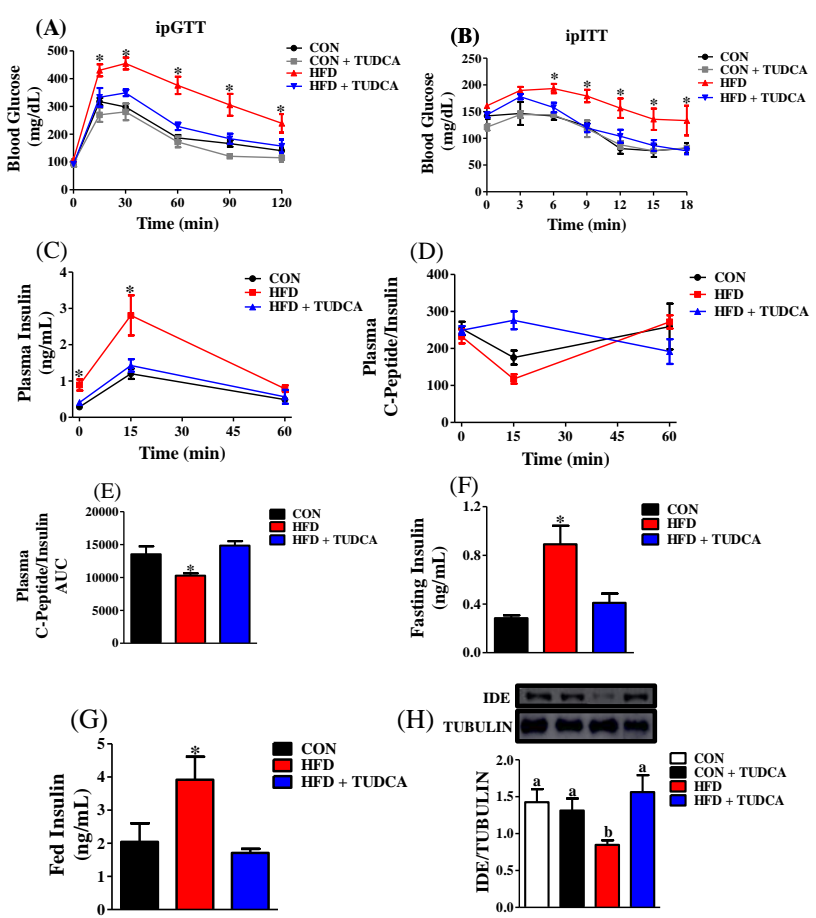

(H) Tubulin

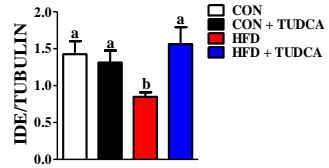

Image 1. GTT (A), ITT (B), insulin (C), C-Peptide (D) plasma levels, C-Peptide/Insulin ration (E), fasting $(F)$ and fed Insulin (G) and liver IDE expression (H) in HFD mice treated or not with TUDCA. Total. Data are mean \pm SEM. $\mathrm{N}=4-6 . t$-test ( ${ }^{*}$ is different from control) or one Way anova, (Different letters indicates statistic differences). P $\leq 0.05$.

\section{Conclusions}

The bile acid TUDCA improves insulin homeostasis, in part, by increasing insulin clearance through IDE expression.

\section{Acknowledgement}

Financial Support: FAPESP/CEPID and CNPq 